\title{
SOME CIRCUMSTANCES WHERE EXTRA UPDATES CAN DELAY MIXING
}

\author{
ALEXANDER E. HOLROYD
}

\begin{abstract}
Peres and Winkler proved a 'censoring' inequality for Glauber dynamics on monotone spins systems such as the Ising model. Specifically, if, starting from a constant-spin configuration, the spins are updated at some sequence of sites, then inserting another site into this sequence brings the resulting configuration closer in total variation to the stationary distribution. We show by means of simple counterexamples that the analogous statements fail for Glauber dynamics on proper colorings of a graph, and for lazy transpositions on permutations, answering two questions of Peres. It is not known whether the censoring property holds in other natural settings such as the Potts model.
\end{abstract}

\section{INTRODUCTION}

Peres and Winkler [6] proved the following striking and useful property of Glauber dynamics on the Ising model. Consider a finite set of sites with arbitrary ferromagnetic pair interactions, and let $\pi$ be the associated stationary distribution on spin configurations. Starting from a constant spin configuration, apply single-site updates at a finite deterministic sequence of sites, where each update consists of replacing the spin at the chosen site with a random spin chosen according to its conditional law under $\pi$ given all other spins. This results in a random configuration. If an additional site is inserted into the update sequence, the resulting configuration is no further from $\pi$ in total variation distance.

The above result has proved to be an invaluable tool in the analysis of mixing time for the Ising model; see the applications in [1, 2, 3, 4, 5]. Peres and Winkler prove their result in the more general setting of monotone spin systems; that is, those in which the set of spins is totally ordered and single-site updates stochastically respect this ordering. The purpose of this note is to demonstrate that analogous

Date: 20 January 2011.

2010 Mathematics Subject Classification. 60J10; 82C20; 05C15.

Key words and phrases. mixing time, censoring, coloring, transposition walk. 
statements fail in two other natural settings: proper colorings and lazy transpositions.

We first consider colorings. Let $G=(V, E)$ be a finite simple graph. A (proper) $q$-coloring of $G$ is a map from $V$ to $\{1, \ldots, q\}$ that assigns distinct values (colors) to adjacent vertices. Let $\mu$ be a probability measure on the set of $q$-colorings and let $v \in V$ be a vertex. Define the recoloring operator $\kappa(v)$ as follows. Let $\mu \cdot \kappa(v)$ be the law of the coloring obtaining from a coloring with law $\mu$ by replacing the color at $v$ with a uniformly random color from the set of colors absent from $v$ 's neighbours (conditional on the existing coloring). Let $\pi$ be the uniform measure on all $q$-colorings of $G$. An equivalent interpretation of $\kappa(v)$ is that the color at $v$ is replaced with a random color chosen according to its conditional law under $\pi$, given the existing coloring of $V \backslash\{v\}$. Observe also that $\pi$ is the stationary distribution of the Markov chain that recolors a random vertex (chosen according to any distribution with full support) at each step.

Proposition 1. Consider proper 4-colorings of the triangle. Let $d$ be any continuous metric on the space of probability measures on colorings, and let $\delta$ be the point measure on some fixed coloring. There exist integers $1 \leq t<m$ and vertices $i_{1}, \ldots, i_{m}, I$ such that, writing

$$
\begin{gathered}
\mu=\delta \cdot \kappa\left(i_{1}\right) \cdots \kappa\left(i_{m}\right), \\
\nu=\delta \cdot \kappa\left(i_{1}\right) \cdots \kappa\left(i_{t}\right) \kappa(I) \kappa\left(i_{t+1}\right) \cdots \kappa\left(i_{m}\right),
\end{gathered}
$$

we have

$$
d(\mu, \pi)<d(\nu, \pi) .
$$

In other words, if, starting from a deterministic coloring, a sequence of vertices is recolored, then inserting an extra vertex in the sequence can move the resulting distribution further from $\pi$. In particular, the result applies when $d$ is total variation distance, in which case it answers a question of Yuval Peres (personal communication).

Now we turn to permutations. Let $\mu$ be a probability measure on the symmetric group of permutations of $V:=\{1, \ldots, n\}$. For a pair $i, j \in V$, we define the lazy transposition operator $\tau(i, j)$ as follows. Let $\rho=(\rho(1), \ldots, \rho(n))$ be a random permutation with law $\mu$, and let $\mu \cdot \tau(i, j)$ be the law of the random permutation obtained from $\rho$ by interchanging $\rho(i)$ and $\rho(j)$ with probability $1 / 2$ (conditional on $\rho$ ), and otherwise leaving $\rho$ unchanged. Let $\delta$ be the point measure on the identity permutation, and let $\pi$ be the uniform probability measure on all $n$ ! permutations. 
Proposition 2. Consider lazy transpositions on $V=\{1, \ldots, 4\}$. Let $d$ be any continuous metric on the space of probability measures on permutations. There exist $m, t, I, J, i_{1}, j_{1}, \ldots$ such that, writing

$$
\begin{gathered}
\mu:=\delta \cdot \tau\left(i_{1}, j_{1}\right) \cdots \tau\left(i_{m}, j_{m}\right) \\
\nu:=\delta \cdot \tau\left(i_{1}, j_{1}\right) \cdots \tau\left(i_{t}, j_{t}\right) \tau(I, J) \tau\left(i_{t+1}, j_{t+1}\right) \cdots \tau\left(i_{m}, j_{m}\right),
\end{gathered}
$$

we have

$$
d(\mu, \pi)<d(\nu, \pi)
$$

\section{Proofs}

We will first prove Proposition 2, and then deduce Proposition 1.

Proof. We will show that, as $(M, N) \rightarrow \infty$,

$$
\delta \cdot[\tau(2,4) \tau(3,4)]^{M} \tau(1,4) \tau(2,4)[\tau(1,4) \tau(3,4)]^{N} \rightarrow \pi,
$$

while

$$
\delta \cdot[\tau(2,4) \tau(3,4)]^{M} \tau(1,4) \tau(3,4) \tau(2,4)[\tau(1,4) \tau(3,4)]^{N} \rightarrow \alpha,
$$

for some $\alpha \neq \pi$. By the continuity of $d$, the required inequality then follows by taking $M$ and $N$ sufficiently large.

We interpret permutations as arrangements of particles, so that in permutation $\mu$, particle $\mu(i)$ is in location $i$, and $\tau(i, j)$ swaps the particles in locations $i, j$ with probability $1 / 2$. Let $\pi_{1}$ be the uniform measure on permutations $\rho$ such that $\rho(1)=1$, and note that

$$
\delta \cdot[\tau(2,4) \tau(3,4)]^{M} \rightarrow \pi_{1} \quad \text { as } \quad M \rightarrow \infty
$$

(by the convergence theorem for irreducible aperiodic Markov chains). Now consider a random permutation $\sigma$ with law

$$
\beta:=\pi_{1} \cdot \tau(1,4) \tau(2,4) .
$$

The location $\sigma^{-1}(1)$ of particle 1 is equal to 2 with probability $1 / 4$, since after the first transposition $\tau(1,4)$ it was 1 or 4 each with probability $1 / 2$. Conditional on the location of particle 1 , the arrangement of particles $2,3,4$ is still uniform, so $\sigma(2)$ (the particle in location 2 ) is exactly uniform among $1, \ldots, 4$. Therefore,

$$
\beta \cdot[\tau(1,4) \tau(3,4)]^{N} \rightarrow \pi \quad \text { as } \quad N \rightarrow \infty,
$$

since conditional on $\sigma(2)$, the effect of the additional transpositions is to uniformize the particles in locations 1,3,4 in the limit. The convergence (11) now follows by the continuity of the transposition operator $\tau(i, j)$.

A similar argument gives (2): after applying the extra transposition $\tau(3,4)$, particle 1 is at location 4 with probability $1 / 4$ (and cannot be at 2$)$, therefore after $\tau(2,4)$ it is at 2 with probability $1 / 8$. Thus 
(2) holds with $\alpha$ the law of some random permutation that has 1 in location 2 with probability $1 / 8$.

Proof of Proposition 1. Let the triangle $G$ have vertices 1,2,3, and assume without loss of generality that $\delta$ is the point measure on the identity map. We may identify a 4-coloring of $G$ with a permutation assigning colors $1, \ldots, 4$ to four vertices $1, \ldots, 4$, with the color at vertex 4 being the one absent from the coloring of $G$. For $i=1,2,3$, the operator $\kappa(i)$ corresponds to the lazy transposition operator $\tau(i, 4)$. Since the example constructed in the proof of Proposition 2 uses only transpositions involving 4 , the same example applies here.

\section{FURTHER REMARKS}

The example in the proof of Proposition 2 was chosen to minimize computations and facilitate the proof of Proposition 1. Naturally, many variations are possible. If $d$ is total variation distance, an explicit computation shows that the required inequality in fact holds with $M=$ $N=1$. As a simpler alternative which does not adapt so readily to coloring, we have

$$
\delta \cdot[\tau(2,4) \tau(3,4)]^{M} \tau(1,4) \tau(2,4) \tau(1,3) \rightarrow \pi, \quad \text { as } \quad M \rightarrow \infty,
$$

while the insertion of $\tau(3,4)$ before the last $\tau(1,3)$ again gives a different limit. Finally, if we relax the problem by allowing a "block update" $\tau(S)$ (defined so as to uniformly permute the elements of a set $S \subset V$ ), then we may of course do away with limits, replacing the expressions []$^{M}$ and [ $]^{N}$ with $\tau(\{2,3,4)\}$ and $\tau(\{1,3,4\})$.

We note that our example adapts to the anti-ferromagnetic Potts model. Consider the 4-state Potts model on a triangle, with antiferromagnetic interactions (i.e. favoring distinct spins) of equal strength $J$ along each edge. As $J \rightarrow \infty$, the transition probabilities for site updates approach those of the 4-coloring model. Hence, starting from a configuration where all 3 vertices have different spins, the example in the proof of Proposition 1 applies here if $J$ is large enough. Moreover, starting from a constant all-1 configuration and updating vertices 2 and 3 results in a configuration that is asymptotically (as $J \rightarrow \infty$ ) uniform on those where vertex 1 has spin 1 . Hence the same example applies with $M=1$.

It is an open question whether extra updates can delay mixing for the ferromagnetic Potts model starting from a constant spin configuration. 


\section{REFERENCES}

[1] J. Ding, E. Lubetzky, and Y. Peres. The mixing time evolution of Glauber dynamics for the mean-field Ising model. Communications in Mathematical Physics, 289(2):725-764, 2009.

[2] J. Ding and Y. Peres. Mixing time for the Ising model: a uniform lower bound for all graphs. 2009, arXiv:0909.5162.

[3] F. Martinelli and A. Sinclair. Mixing time for the solid-on-solid model. In Proceedings of the 41st annual ACM symposium on Theory of computing, pages 571-580. ACM, 2009.

[4] F. Martinelli and F. Toninelli. On the mixing time of the 2D stochastic Ising model with plus boundary conditions at low temperature. Communications in Mathematical Physics, 296(1):175-213, 2010.

[5] E. Mossel and A. Sly. Exact Thresholds for Ising-Gibbs Samplers on General Graphs. 2009, arXiv:0903.2906.

[6] Y. Peres and P. Winkler. Can extra updates delay mixing:11 Preprint.

Microsoft Research, 1 Microsoft Way, Redmond, WA 98052, USA

E-mail address: holroyd at microsoft.com

$U R L:$ http://research.microsoft. com/〜holroyd

\footnotetext{
${ }^{1}$ Yes.
} 